\title{
ОБЗОР ХИРУРГИЧЕСКОГО ЛЕЧЕНИЯ ПЕРВИЧНЫХ ОПУХОЛЕЙ ЗАБРЮШИННОГО ПРОСТРАНСТВА
}

\section{REVIEW OF SURGICAL TREATMENT FOR PRIMARY RETROPERITONEAL TUMORS}

\section{Timerbulatov \\ E. Senderovich F. Irnazarov \\ E. Grishina \\ L. Aitova \\ T. Ziganshin}

Summary. Primary Retroperitoneal Tumors are found in $0.03-1.1 \%$ of all neoplasms, more than half of them are malignant, in $50 \%$ of cases within 12-18 months after radical surgical treatment, a relapse occurs. Most often, these tumors are found in young and middle-aged women.

To determine the tactics of treatment and the choice of the type of surgical intervention, it is necessary to conduct a preliminary study with obligatory histological examination.

A minimally invasive method for the treatment of primary retroperitoneal tumors can reduce the duration of hospitalization and pain period in the most cases

Mini-invasive approaches when removing the primary retroperitoneal tumors are associated with less trauma to the patient, a shorter pain period, faster recovery, and a short hospitalization period. However, a serious obstacle for laparoscopic or robotic-assisted tumor removal is late diagnosis, when the size of the tumor does not allow the operation through the mini-access.

For successful treatment with a mini-invasive approach, it is necessary to diagnose a tumor up to $7-10 \mathrm{~cm}$ in size; nowadays, such tumors are most often diagnosed for the first time when they reach $20 \mathrm{~cm}$.

Keywords: primary retroperitoneal tumors, retroperitoneal tumors, laparoscopic surgery, Robot-assisted surgery, surgery treatment, transperitoneal approach, retroperitoneal approach.
Тимербулатов Махмуд Вилевич

Д.м.н., профессор, ФГБОУ ВО «Башкирский государственный медицинский университет»

Минздрава России (Уфа) timerm@yandex.ru

Сендерович Ефим Иосифович

К.м.н., дочент ФГБОУ ВО «Башкирский государственный медицинский университет» Минздрава России(Уфра) efim.senderovich@mail.ru

Ирназаров Фагим Ринатович Ассистент, аспирант, ФГБОУ ВО «Башкирский государственный медицинский университет» Минздрава России(Уфа) fm.irnazarov@gmail.com

Гришина Елена Евгеньевна

К.м.н., доцент, ФГБОУ ВО «Башкирский государственный медицинский университет» Минздрава России (Уфра) alyonagrishina662@mail.ru

Аитова Лилия Ринатовна

К.м.н., доцент, ФГБОУ ВО «Башкирский государственный медицинский университет»

Минздрава России (Уфа)

liaitova@yandex.com

Зиганиин Тимур Маратович К.м.н., ГБУЗ РБ ГКБ № 21 (Уфра) tim.zig2012@yandex.ru

Аннотация. Неорганные забрюшинные опухоли встречаются 0,03-1,1\% случаев всех новообразований, более половины из них - злокачественные, в 50\% случаев в течение 12-18 месяцев после радикального хирургического лечения происходит рецидив. Чаще данные опухоли встречаются у женщин молодого и среднего возраста.

Для определения тактики лечения и выбора вида хирургического вмешательства необходимо провести исследование с обязательным гистологическим исследованием.

Мини-инвазивный способ лечения неорганных забрюшинных опухолей позволяет снизить длительность госпитализации, болевой период и травматизацию пациента в большинстве случаев

Мини инвазивные доступы при удалении Н30 ассоциированы с меньшей травматизацией пациента, более коротким болевым периодом, быстрым выздоровлением и непродолжительным периодом госпитализации. Однако, серьезным препятствием для лапароскопического или робот-ассистированого удаления опухоли является поздняя диагностика, когда размеры опухоли не позволяют провести операцию через мини-доступ. 


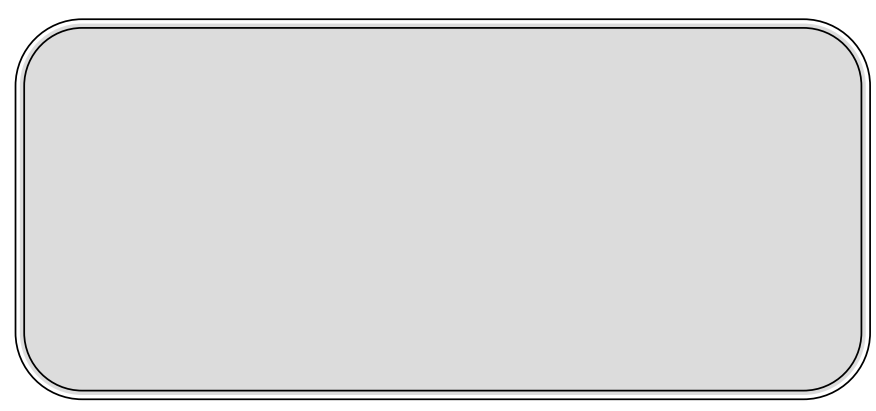

\section{Актуа^ьность}

$\mathbf{H}$ е имеющие органной принадлежности опухоли, локализующиеся забрюшинно между листками брюшины брыжеек кишечника объединены в понятие неорганных забрюшинных опухолей (Н3O). Частота их встречаемости составляет 0,03-1,1\% от всех впервые выявленных новообразований, при этом $60-85 \%$ Н3О являются злокачественными, рецидивирующими в 50\% (по данным некоторых исследование - до 61\%) случаев в течение 12-18 месяцев после радикального хирургического лечения $[1,2,3,4]$.

Чаще данные опухоли встречаются у женщин любого возраста, но преимущественно 40-60 лет $[5,10,16,26]$

Морфологически согласно классификации опухолей Всемирной организации здравоохранения (ВО3) Н3О могут быть представлены мезенхимальными опухолями (включая саркомы и нейрогенные опухоли) парасимпатическими, лимфоидными опухолями, экстрагонадными опухолями зародышевых клеток. По данным различных исследований в большинстве случаев Н3О представлены различными формами сарком.[51]

Стратегии лечения значительно отличаются для различных типов новообразования, поэтому необходимо иметь точный диагноз перед началом лечения.

Диагностика Н3О может быть затруднена из-за часто бессимптомного течения, так же возможны сложности в верификации опухоли из-за ее расположения (новообразование, кажущееся исходящим из надпочечников, на самом деле может быть опухолью, расположенной вокруг органа). [28,29,30,31,43,46]. Анамнез и физикальное обследование могут помочь установить диагноз (например, пациент с раком яичка имеет высокие риски поражения забрюшинного пространства. Однако большинство НЗО не имеют очевидного диагноза, требуют тщательной диагностики (исследование онкомаркеров, проведение биопсии, КТ/МРТ исследований). Если онкомаркеры отрицательные, необходимо провести предоперационную чрезкожную биопсию для определения или подтверждения диагноза. Проведенные
Для успешного проведения лечения мини-инвазивным доступом необходимо диагностировать опухоль размером до 7-10 см, в настоящее время чаще всего такие опухоли впервые диагностируются при достижении $20 \mathrm{~cm}$.

Ключевые слова: неорганные забрюшинные опухоли, забрюшинные опухоли, лапароскопическая операция, робот-ассистированная хирургия, хирургическое лечение, трансперитонеальный доступ, ретроперитонеальный доступ.

исследования показывают, что пункционная биопсия безопасна и не увеличивает частоту рецедивов злокачественных новообразований. [27, 31,43,46].

Основным методом лечения первичных и рецидивных неорганных забрюшинных опухолей является хирургическое вмешательство. Лучевая терапия и химиотерапия мало эффективны, хотя могут использоваться при комбинированном лечении Н3О либо как самостоятельные методы при невозможности удаления опухоли. Поэтому пути совершенствования тактики хирургического лечения остаются актуальными $[9,11,15]$.

Сложность хирургического лечения Н3О связана с отсутствием топографических ориентиров и ограниченным пространством забрюшинной области, трудность определения наличия инвазии опухоли в магистральные сосуды и расположенные рядом органы, визуализации питающих опухоль сосудов.

На данный момент не существует единой классификации локализации НЗО. В.В. Цвиркуном в 2000 г в институте хирургии им. А.В. Вишневского была разработана классификация, основывающаяся на выделении 5 зон забрюшинного пространства (рис. 1):

1 - между левым куполом диафрагмы сверху, супраренальным сегментом Наличие образования в зоне оценивается по шкале от 0 до 5. Если зона свободна от образования, ставится 0 [2]

Другие авторы исследующие проблему хирургического лечения $\mathrm{H} 30$ разрабатывают собственных схемы (рис 2)

Хирургическое лечение больных с диагнозом Н3О сопровождается высоким риском интраоперационных и послеоперационных осложнений. Для радикального удаления забрюшинных опухолей необходима длительная ретракция органов брюшной полости, адекватная экспозиция, сложность проведения операции составляет так же необходимость проведения хирургических манипуляций к магистральным сосудам и нервным структурам $[1,2,3,6,7,8,14,17,18,19]$ 


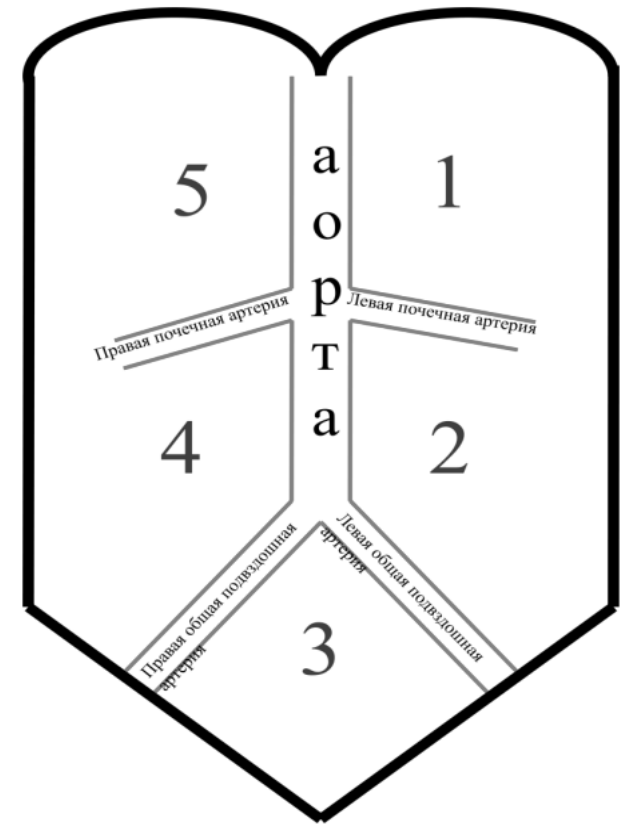

Рис 1. Классификации локализации Н3О по В.В. Цвиркуну*

*рисунок Сон.А.И. Робот-ассистированные операции при неорганных забрюшинных образованиях 2017 г [4]

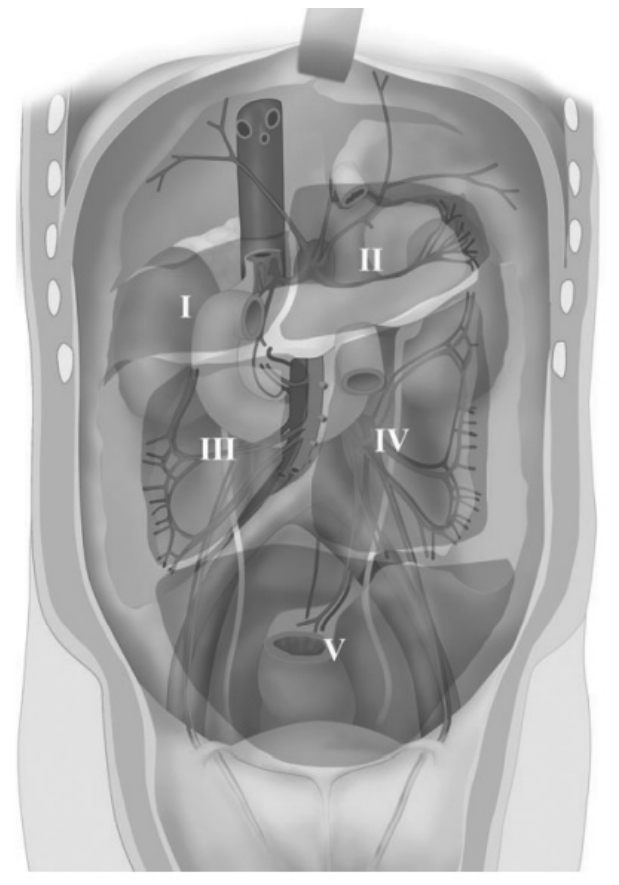

Рис 2. Схема локализации H3O Wei Ding, MD**

** рисунок Wei Ding, MD и авт. Minimally Invasive Surgery of Primary Retroperitoneal Tumors from the Perspective of General Surgeons: 6 Years of Experience at a Single Institution 2020[24]
В связи с этим проводятся исследования в поиске оптимальной хирургической техники удаления Н3О. Мы изучили результаты исследований, проводимых различными клиническими центрами, сравнивающими лапаротомную, лапароскопическую и робот-ассистированную техники оперативного вмешательства

По результатам анализа ретроспективных исследований для успешного проведения лапароскопической или робот-ассистированной операции размер новообразования не должен превышать 7-10 см (при большем размере требовалась конверсия), в тоже время средний размер Н3О при постановке диагноза составляет 20 см. [4,20, 21, 22,23,24,25,32]

Поскольку чаще Н3О располагаются у задней брюшной стенки, при лапаротомической операции требуется широкий доступ, что удлиняет время послеоперационного восстановления.

Длительность операции короче при открытом доступе (медиана 112 мин), при лапароскопическом доступе -медиана 125, робот-ассистированные (РА)операции медиана 140 мин.
Объем кровопотери при открытом доступе в среднем в 2,5 раза выше, аналогичного показателя для лапароскопии или робот-ассистированных операций, что связывают с большим размером опухоли, следовательно с увеличением сложности и травматизма операции. Нахождение пациентов стационаре при мини инвазивных вмешательствах было короче (4-6 дней для робот-ассистированного вмешательства, 5-7 дней для лапароскопий и 8-10 дней для открытого доступа) [1,2,3,37,24]

Анализ ретроспективных парных исследований за период с 2006 по 2016 гг., в которых между парами пациентов сравнивались трансперитонельный и ретроперитонеальные доступы при радикальном лечении Н3О, показал что ретроперитонеальный доступ статистически значимо снижает запланированную кровопотерю и время операции. Послеоперационный исходы для данных операционных доступов эквивалентны [33, 34, 35, 38,39, 40,42,45,47,48,49]

В представленных исследованиях послеоперационные осложнения (кровотечения, лимфорея, нагноение послеоперационной раны) не коррелируют с выбором операционного доступа $[12,13,36,37,41,44,50]$ 


\section{Зак^ючение}

Мини инвазивные доступы при удалении $\mathrm{H} 30$ ассоциированы с меньшей травматизацией пациента, более коротким болевым периодом, быстрым выздоровлени- ем и непродолжительным периодом госпитализации. Однако, серьезным препятствием для лапароскопического или робот-ассистированого удаления опухоли является поздняя диагностика, когда размеры опухоли не позволяют провести операцию через мини-доступ.

\section{ЛИТЕРАТУРА}

1. Ближайшие и отдаленные результаты лечения больных с опухолями забрюшинного пространства /0.И. Каганов [и др.] // Поволжский онкологический вестник. 2020. № 3. С. 12-19

2. Ближайшие и отдаленные результаты лечения опухолей забрюшинного пространства /Каганов 0.И. [и др.]// Proceedings of the First International Forum of Oncology and Radiology. 2019. C. 130

3. Хирургическое лечение неорганных забрюшинных опухолей /А.Г. Кригер [и др.]// Хирургия. 2017. № 1. С. 15-20.

4. А.И. Сон Робот-ассистированные операции при неорганных забрюшинных образованиях: дис. ... канд. мед наук: 14.01.17.— М., 2017. 123с.

5. Lam A.K. Update on adrenal tumours in 2017 Whorld Health Organization (WHO) of endocrine tumours.// Endocrine Pathology. 2017. № 28. P. 213-277

6. Adrenal tumours: open surgery versus minimally invasive surgery/ Cristian Fiori [et al]// Current opinion in oncology. 2020. № 32(1). P. 27-34

7. Algorithm for the surgical management of mesenchymal tumors of the perineum / Sourrouille I [et al]// Diseases of the colon and rectum.2015. № 58(3). P. 304-313

8. Alternative approaches to retroperitoneal lymph node dissection for paratesticular rhabdomyosarcoma/ Sara A Mansfield [et al]// Journal of pediatric surgery.2020. № 50(12). P. 2677-2681.

9. A phase III randomized study of preoperative radiotherapy plus surgery versus surgery alone for patients with retroperitoneal sarcoma/ Bonvalot S [et al]// Journal of Clinical Oncology. 2019. № 15. P. 264-265

10. A retroperitoneal mass needs respect/ Royal Australasian College of Surgeons// ANZ journal of surgery. — 2018.-№ 88(4).P.261-262

11. Benefit of the Surgical Management of Retroperitoneal Sarcoma in a Reference Center: A Nationwide Study of the French Sarcoma Group from the NetSarc Database/ Bonvalot S [et al]// Annals of Surgical Oncology. 2019. № 26(7). P. 2286-2293

12. Clinical predictors of prolonged postresection hypotension after laparoscopic adrenalectomy for pheochromocytoma/ TakeshiNamekawaMD [et al]// Surgery. 2016. N159(3). P. 763-770

13. Current principles of surgery for retroperitoneal sarcomas/ Fairweather M [et al]// Journal of surgical oncology. — 2018.-№ 117(1). P. 33-41

14. Hemodynamic instability during surgery for pheochromocytoma: comparing the transperitoneal and retroperitoneal approach in a multicenter analysis of 341 patients/ Wessel M C M Vorselaars [et al]// Surgery.2018. № 163(1). P. 176-182

15. Improved survival using specialized multidisciplinary board in sarcoma patients /Blay J [et al]//Annals of Oncology.2017 № 28(11). P. 2852-2859

16. Soft tissue and visceral sarcomas: ESMO-EURACAN clinical practice guidelines for diagnosis, treatment and follow-up/ Casali PG [et al]// Annals of Oncology. 2018.№ 29. P. 51-67

17. Interdisciplinary approach allows minimally invasive, nerve-sparing removal of retroperitoneal peripheral nerve sheath tumors/ Mohammed Mehdi Hajiabadi [et al]// Langenbeck's archives of surgery. 2020. № 405(2). P. 199-205

18. Laparoscopic adrenalectomy using the lateral reproperitoneal approach: is it a safe and feasible treatment option for pheochromocytomas larger than $6 \mathrm{~cm}$ ? I Chung HS [et al] // International Journal of Urology. 2018.№ 25. P. 414-419

19. Laparoscopic anterior versus endoscopic posterior approach for adrenalectomy: a shift to a new golden standard?/ 0.M. Vrielink [et al]// Langenbeck's Archives of Surgery. 2016. № 402. P. 767-773

20. Lateral transperitoneal adrenalectomy versus posterior retroperitoneoscopic adrenalectomy for benign adrenal gland disease: randomized controlled trial at a single tertiary medical center / Chai YJ [et al] Annals of Surgery. 2019. № 269(5). P. 842-848

21. Lower pole approach in retroperitoneal laparoscopic radical nephrectomy: a new approach for the management of renal vascular pedicle/ Bo Yuan [et al]// World journal of surgical oncology. 2018. № 16(1). P. 31

22. Management of metastatic retroperitoneal sarcoma: a consensus approach/Trans-Atlantic Retroperitoneal Sarcoma Working Group (TARPSWG)// Annals of Oncology.2018.№ 29(4). P. 857-871

23. Minimally Invasive Lateral Retroperitoneal Approach for Resection of Extraforaminal Lumbar Plexus Schwannomas: Operative Techniques and Literature Review/ Michael M Safaee [et al]// Opererative Neurosurgery.2018. № 15(95). P. 516-521

24. Minimally Invasive Surgery of Primary Retroperitoneal Tumors from the Perspective of General Surgeons: 6 Years of Experience at a Single Institution/ Wei Ding, MD [et al]// Journal of laparoendoscopic \& advanced surgical techniques. 2020.№ 31(6). P. 698-704

25. National trends and disparities of minimally invasive surgery for localized renal cancer, 2010 to 2015/ Xia L [et al]// Urologic Oncolgy. 2019.№ 37(3). P. 182e17.$182 \mathrm{e} 27$

26. Overview of primary adult retroperitoneal tumours /Improta [et al]// European Journal of Surgical Oncology.2020.№ 46(9).P. 1773-1579

27. Percutaneous core needle biopsy in retroperitoneal sarcomas does not influence local recurrence or overall survival/ Wilkinson MJ [et al]//Annals of surgical oncology. 2015.№ 22(3).P. 853-858 
28. Primary retroperitoneal soft tissue sarcoma: imaging appearances, pitfalls and diagnostic algorithm /Messiou C[et al]// European journal of surgical oncology.2017.№ 43. P. 1191-1198

29. Renal angiomyolipomas: At least two diseases. A series of patients treated at two European institutions/ Delhorme JB [et al]// European journal of Surgical Oncology.2017.№ 43(4). P. 831-836

30. Renal mass and localized renal cancer: AUA guideline / Campbell S [et al]// Journal of Urology.2017 № 198(3).P. 520-529

31. Retroperitoneal Sarcomas: Does Laterality Matter?/ Johanna Lou [et al]// Journal of surgical research. 2019.№ 41.P. 34-41

32. Retroperitoneoscopic approach for highly complex posterior renal hilar tumors/ Jose Luis Bauza [et al]// Official journal of the Brazilian Society of urology. 2020.№ 46(3).P.485-486

33. Retroperitoneal approach for robot-assisted partial nephrectomy: technique and early outcomes/ Porreca A [et al]// Official journal of the Brazilian Society of urology. 2018.№ 44(1).P. 63-68

34. Retroperitoneal vs Transperitoneal Robot-assisted Partial Nephrectomy: Comparison in a Multi-institutional Setting/ Sohrab Arora [et al]// Urology.2018. № 120. P. 131-137

35. Retroperitoneal Versus Transperitoneal Robotic Partial Nephrectomy: A Multicenter Matched-pair Analysis/ Nina N Harke [et al]// European urology focus. 2020 № 20. P. 8

36. Risk factors for complications after robotic adrenalectomy: a review/ Davide Inversini [et al]//Gland surgery. 2020. № 9(3). P. 826-830

37. Robot-Assisted Laparoscopic Excision of Complicated Retroperitoneal Tumors with Four Arms Via Retroperitoneal Way: A Unique Minimal-Invasive Approach / Sunyi Ye [et al]// Journal of 0 laparoendoscopic\&advanced surgical techniques.2019.№ 30(10).P. 1110-1116

38. Selektionskriterien zur laparoskopischen oder retroperitoneoskopischen Adrenalektomie/ Henning Dralle [et al]// Springer Nature. 2020.№ 91 (7).P.595

39. Robotic Assisted Retroperitoneal Lymph Node Dissection for Small Volume Metastatic Testicular Cancer/ Andreas Hiester [et al]// The journal of urology.2020. № 204(6).P.1242-1248

40. Robotic partial nephrectomy for posterior tumors through a retroperitoneal approach ofers decreased length of stay compared with the transperitoneal approach: a propensitymatched analysis / Maurice MJ [et al]// Journal of Endourology.2017.№ 31(2).P. 158-162

41. Role of nutritional status in the early postoperative prognosis of patients operated for retroperitoneal liposarcoma (RLS): A single center experience/Kirov KM [et al] European journal of surgical oncolology.2019.№ 45(2).P. 261-267

42. Selective Versus Non-selective a-Blockade Prior to Laparoscopic Adrenalectomy for Pheochromocytoma/ Reese W. Randle MD [et al]// Annals of Surgical Oncology. 2017. № 24. P. 244-250

43. Soft tissue sarcoma, Version 2.2018, NCCN clinical practice guidelines in oncology/ Von Mehren M [et al]// Journal of the National Comprehensive Cancer Network: JNCCN. — 2018.-№ 16(5). P. 536-563

44. Surgery for Abdominal Well-Differentiated Liposarcoma/Sara A Mansfield [et al]//Current treatment options in oncology.2018. № 19(1). P. 1

45. Surgical management of primary retroperitoneal sarcomas: rationale for selective organ resection / Fairweather M [et al]// Annals of surgical oncology.2018 № 25(1). P. 98-106

46. The management of desmoid tumours: A joint global consensus based guideline approach for adult and paediatric patients./Desmoid Tumor Working Group// European journal of Cancer.2020.№ 127. P. 96-107

47. Transperitoneal versus retroperitoneal robot-assisted partial nephrectomy: a systematic review and meta-analysis/ Xia L [et al]// International Journal of Surgery.2016.№ 30. P. 109-115

48. Transperitoneal versus retroperitoneal laparoscopic adrenalectomy for large pheochromocytoma: Comparative outcomes/ Koji Shiraishi [et al]// international journal of urology. 2019. № 26(2). P. 212-216

49. Transperitoneal vs. retroperitoneal robotic partial nephrectomy: a matched-paired analysis/ Harsha R. Mittakanti [et al]//World Journal of Urology.2019№ 38(5). P. 1093-1099

50. Treatment at low-volume hospitals is associated with reduced short-term and long-term outcomes for patients with retroperitoneal sarcoma/ Keung EZ [et al]// Cancer.2018. № 124(23). P. 4495-4503

51. WHO Classification of Tumours Online // URL: https://tumourclassification.iarc.who.int/welcome/ (дата обращения: 16.08.2021).

\footnotetext{
๑ Тимербулатов Махмуд Вилевич ( timerm@уаndex.ru ), Сендерович Ефим Иосифович ( efim.senderovich@mail.ru ), Ирназаров Фагим Ринатович ( fm.irnazarov@gmail.com ), Гришина Елена Евгеньевна ( alyonagrishina662@mail.ru ), Аитова Лилия Ринатовна ( liaitova@yandex.com ), Зиганшин Тимур Маратович ( tim.zig2012@yandex.ru ). Журнал «Современная наука: актуальные проблемы теории и практики»
} 